\title{
Chromogranin-A Expression as a Novel Biomarker for Early Diagnosis of Colon Cancer Patients
}

\author{
Xueli Zhang ${ }^{1,2}{ }^{(\mathbb{D}}$, Hong Zhang ${ }^{1, *(1)}$, Bairong Shen ${ }^{2, *}$ and Xiao-Feng Sun ${ }^{3, *}$ \\ 1 School of Medicine, Institute of Medical Sciences, Örebro University, SE-70182 Örebro, Sweden; \\ zhang.xueli@oru.se \\ 2 Centre for Systems Biology, Soochow University, Suzhou 215006, China \\ 3 Department of Oncology and Clinical and Experimental Medicine, Linköping University, \\ SE-58183 Linköping, Sweden \\ * Correspondence: hong.zhang@oru.se (H.Z.); bairong.shen@suda.edu.cn (B.S.); xiao-feng.sun@liu.se (X.-F.S.); \\ Tel.: +46-19301302 (H.Z.); +86-52165110951 (B.S.); +46-101032066 (X.-F.S.)
}

Received: 9 May 2019; Accepted: 12 June 2019; Published: 14 June 2019

\begin{abstract}
Colon cancer is one of the major causes of cancer death worldwide. The five-year survival rate for the early-stage patients is more than $90 \%$, and only around $10 \%$ for the later stages. Moreover, half of the colon cancer patients have been clinically diagnosed at the later stages. It is; therefore, of importance to enhance the ability for the early diagnosis of colon cancer. Taking advantages from our previous studies, there are several potential biomarkers which have been associated with the early diagnosis of the colon cancer. In order to investigate these early diagnostic biomarkers for colon cancer, human chromogranin-A (CHGA) was further analyzed among the most powerful diagnostic biomarkers. In this study, we used a logistic regression-based meta-analysis to clarify associations of CHGA expression with colon cancer diagnosis. Both healthy populations and the normal mucosa from the colon cancer patients were selected as the double normal controls. The results showed decreased expression of CHGA in the early stages of colon cancer as compared to the normal controls. The decline of CHGA expression in the early stages of colon cancer is probably a new diagnostic biomarker for colon cancer diagnosis with high predicting possibility and verification performance. We have also compared the diagnostic powers of CHGA expression with the typical oncogene KRAS, classic tumor suppressor TP53, and well-known cellular proliferation index MKI67, and the CHGA showed stronger ability to predict early diagnosis for colon cancer than these other cancer biomarkers. In the protein-protein interaction (PPI) network, CHGA was revealed to share some common pathways with KRAS and TP53. CHGA might be considered as a novel, promising, and powerful biomarker for early diagnosis of colon cancer.
\end{abstract}

Keywords: CHGA; colon cancer; biomarker; early diagnosis; logistic regression; meta-analysis; PPI

\section{Introduction}

Colon cancer is one of the leading causes of cancer death worldwide [1]. In 2018, there were more than 1,096,601 new diagnosed colon cancer cases, and around 551,269 patients were dead from the colon cancer worldwide [2]. The advanced modern medicines and surgery techniques have nowadays benefited the stage I and II colon cancer patients and their five-year survival rate has reached to $90 \%$ [3]. However, the five-year survival rate for the stage IV colon cancer patients is only around $10 \%$ [3]. Moreover, more than $50 \%$ of colon cancer patients are already at the late stages when they are clinically diagnosed [4]. As such, it is urgent to find more convenient and accurate biomarkers for early diagnosis of colon cancer.

Although colonoscopy and biopsy pathological examination have been considered as the golden test for colon cancer final diagnosis and primary treatment [5], there are a number of early colon cancer 
cases which do not reveal the typical characteristics, making it difficult to make a clear diagnosis. Accumulating studies on the biomarkers in colon cancer diagnosis have partially solved the problem [6]. However, it is necessary to find more precise biomarkers which can complement the golden test for the early diagnosis of colon cancer.

Biomarkers as biological indicators and conditions have been widely believed to improve the diagnosis, therapeutic response, and prognosis of colon cancer [5]. Thus, many researcher groups, including ours, have been putting a lot of effort into identifying the significance of various biomarkers for colon cancer [7-10]. In our previous work, we established an integrated biomarker database for colorectal cancer (CRC): CBD (http://sysbio.suda.edu.cn/CBD/index.html), in which we collected and summarized the ontology-based biomedicine information from all the published CRC biomarkers up to 2018 [11]. In the CBD, we recorded 62 biomarkers concerning the diagnosis for early-stage colon cancer. However, none of such biomarkers have been identified as ideal clinical applications. Hence, it is markedly important to further explore new promising biomarkers concerning early diagnosis, therapeutic response, and prognosis for CRC.

Human chromogranin-A (CHGA), as a 439-residue-long protein in the secretory granules of many normal and neoplastic neuroendocrine cells, plays a major role in the protein co-stored and co-released with catecholamines from secretory vesicles in adrenal medulla and postganglionic sympathetic axons [12,13]. Expression of CHGA has been proven to be associated with prognosis in CRC [14-16], and has been currently accepted as the main biomarker for neuroendocrine neoplasms $[17,18]$. Recently, CHGA has been approved as an early diagnosis biomarker for gastric cancer [19], prostate cancer [20], and pancreatic neuroendocrine tumors [21]. However, there is no study concerning CHGA expression and early diagnosis in colon cancer.

KRAS as a typical oncogene for cancer initiation and progression has been proven in many cancer types, including CRC, with strong impact on early diagnosis, cancer progression, and prognosis [22]. There are up to $50 \%$ of CRC patients with KRAS gene mutations in their early stages, and half of such patients are unlikely to benefit from the antibody therapy [22]. TP53 as a classic tumor suppressor has revealed more than $60 \%$ of CRC patients with TP53 mutations, which is one of the commonest genetic events in the development of human CRC [23]. The MKI67 is a biomarker of cellular proliferation and has been reported as an independent index for CRC cell growth [24]. The expression of MKI67 has been gradually increased from normal tissue, adenomas to adenocarcinomas, in CRC patients. The overexpression of MKI67 has been considered as the significant biomarker and predictor for primary CRC screening [25].

Machine learning has been widely utilized in bioinformatics for screening potential biomarker candidates and their complex molecular networks [26]. The protein-protein interaction (PPI) network has been proven with high confidence in biomedicine research [27]. In a previous study, we utilized the support vector machine (SVM) and regression tree to predict new potential colon cancer biomarker based on the PPI network, with high indication that CHGA expression, among several others, could be a promising significant biomarker for early diagnosis of colon cancer [28]. To our knowledge, there is no report concerning the association of the CHGA with early diagnosis of colon cancer, although it is necessary to be further verified.

In this study, we have used logistic regression-based meta-analysis, a quantitative review method to detect the diagnostic value of different biomarkers in medical researches [29], and a huge number of gene expression (GE) data for different populations from the Gene Expression Omnibus (GEO) database, as a well-known biomedicine database, to investigate the diagnostic value of CHGA expression as a biomarker, compared to the healthy populations and the normal mucosa from the colon cancer patients. We showed that CHGA expression might be considered as a novel biomarker for early diagnosis of colon cancer, as compared to most well-studied expressions of KRAS, TP53, and MKI67. 


\section{Results}

\subsection{Logistic Regression-Based Meta-Analysis Has Been Used in This Study}

The Study Design Was Represented in Figure 1.

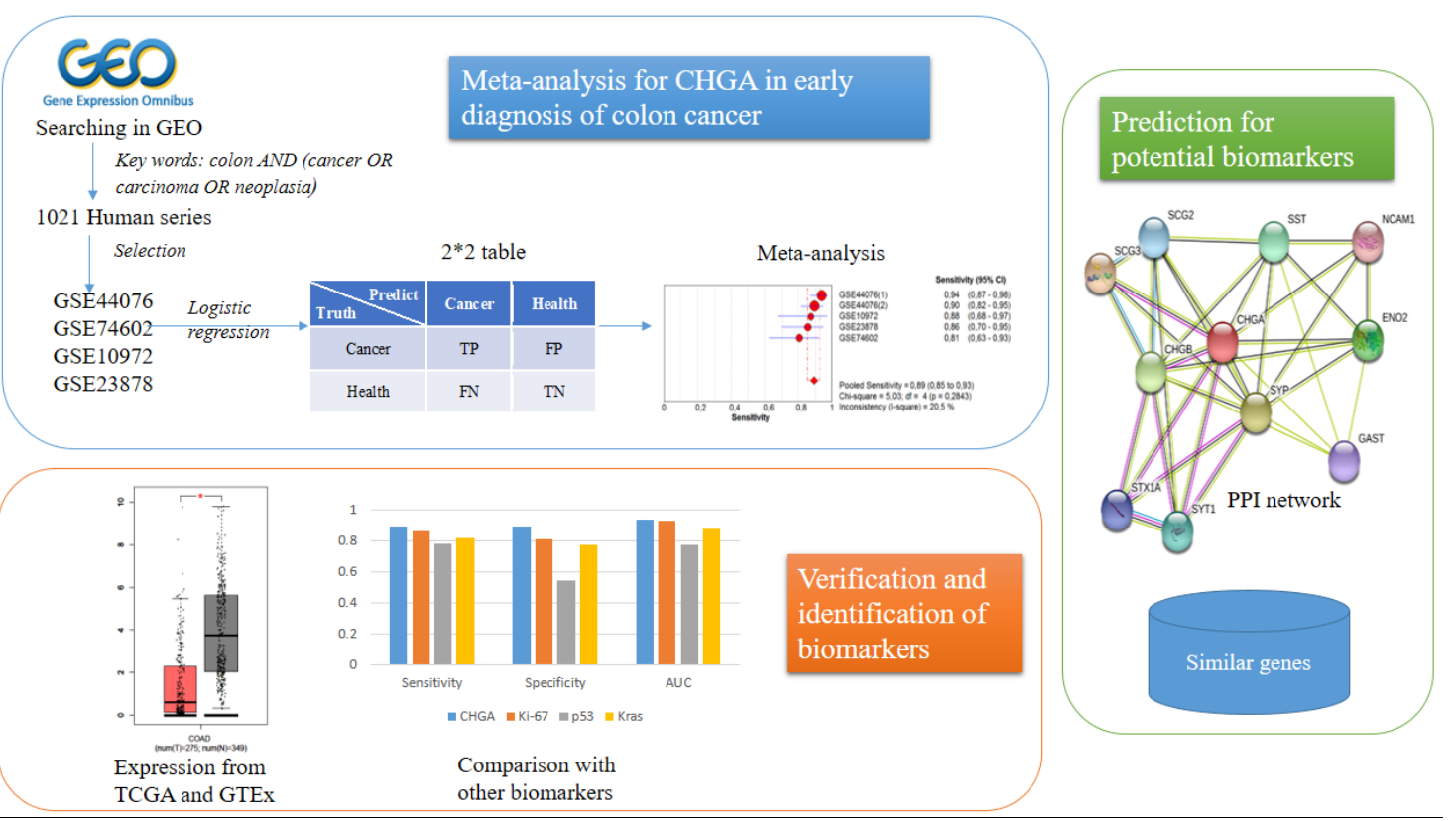

Figure 1. Study pipeline. In this study we used the human microarray Gene expression (GE) data from the Gene Expression Omnibus (GEO) database and logistic regression to formulate the $2 \times 2$ table for meta-analysis. After the chromogranin-A (CHGA) diagnostic meta-analysis, we utilized the RNA-seq data from The Cancer Genome Atlas (TCGA) and Genotype-Tissue Expression (GTEx) to test the CHGA expression in colon cancer patients and healthy controls to verify the results for meta-analysis. Meanwhile the diagnostic meta-analysis for several other reported biomarkers were also conducted using the same datasets. Finally, we predicted several CHGA-associated biomarkers from the protein-protein interaction (PPI) network and GE level.

\subsection{Data Collection}

Microarray human series were collected from the GEO database using a keywords search. There were 1021 GEO datasets collected and 1012 were excluded due to lack of stage information. There were two datasets without detailed cancer patients and experiments information. One dataset did not contain enough samples and two datasets did not include the GE data for CHGA. Eventually, there were four ideal datasets (GSE44076, GSE74602, GSE10972, and GSE23878), including 187 colon cancer patients and 226 normal controls for the analyses. Table 1 shows the characteristics of the included studies.

Table 1. Characteristics of studies of primary colon cancers (PCC) included in the meta-analysis.

\begin{tabular}{llllllll}
\hline Datasets & Sample Number & Stage & Region & Source & Expression & Platform & PMID $^{*}$ \\
\hline GSE44076 & $98 / 148$ & PCC & Barcelona & Tissue & Array & GPL13667 & 25215506 \\
GSE74602 & $30 / 30$ & PCC & Singapore & Tissue & Array & GPL6104 & NA \\
GSE10972 & $24 / 24$ & PCC & Singapore & Cell & Array & GPL6104 & 18538736 \\
GSE23878 & $35 / 24$ & PCC & Riydh & Tissue & Array & GPL570 & 21281787 \\
\hline
\end{tabular}

* Sample number: Patient/Control; PMID: PubMed ID. 


\subsection{Logistic Regression}

Logistic regression was utilized to clarify the expression data to establish the $2 \times 2$ table for meta-analysis (Table 2). GSE44076 contains 2 groups of control: one from healthy samples, the other one from corresponding normal adjacent mucosa of patients.

Table 2. Logistic regression results $(2 \times 2$ table $)$.

\begin{tabular}{lcccc}
\hline \multicolumn{1}{c}{ Datasets } & TP & FP & FN & TN* $^{*}$ \\
\hline GSE44076 (1) & 92 & 1 & 6 & 49 \\
GSE44076 (2) & 87 & 5 & 10 & 93 \\
GSE74602 & 25 & 5 & 6 & 24 \\
GSE10972 & 21 & 10 & 3 & 14 \\
GSE23878 & 30 & 3 & 5 & 21 \\
\hline
\end{tabular}

* TP: True positive; FP: False positive; FN: False negative; TN: True negative.

\subsection{CHGA in the Diagnosis of Early-Stage Colon Cancer}

Figure 2 presents the results, using forest plots, from the meta-analysis. Figure 2A shows the sensitivity of the forest plot for CHGA as a biomarker in the diagnosis of early-stage colon cancer, from which a pooled sensitivity of 0.89 (0.85 to 0.93$)$ was calculated. The specificity forest plot was drawn in Figure 2B $(0.89,0.85$ to 0.93$)$. Figure $2 \mathrm{C}, \mathrm{D}$ present the likelihood ratios for CHGA: positive-likelihood ratio (PLR) 7.86 (2.27 to 27.25) and negative-likelihood ratio (NLR) 0.14 (0.08 to 0.22). CHGA performed a diagnostic odds ratio (DOR) of 57.27 (14.83 to 222.24), which is available on the forest plot in Figure 2E. All these statistics were based on 95\% confidence.

A
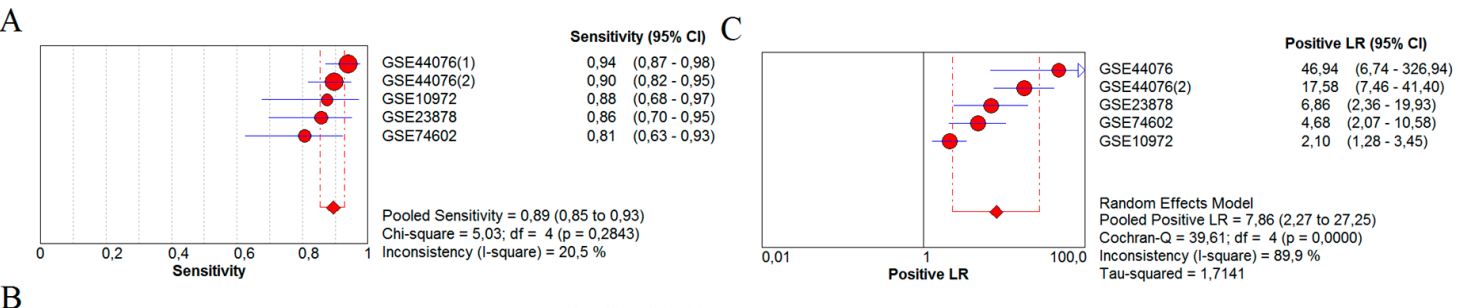

B
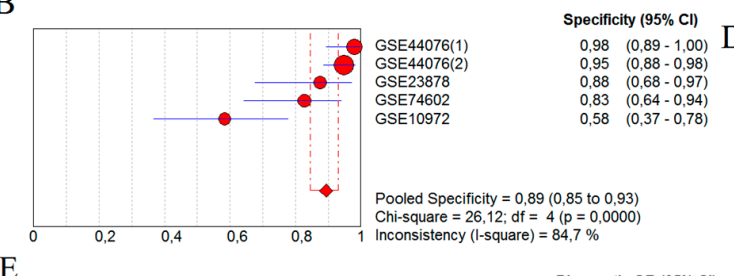

$\mathrm{E}$
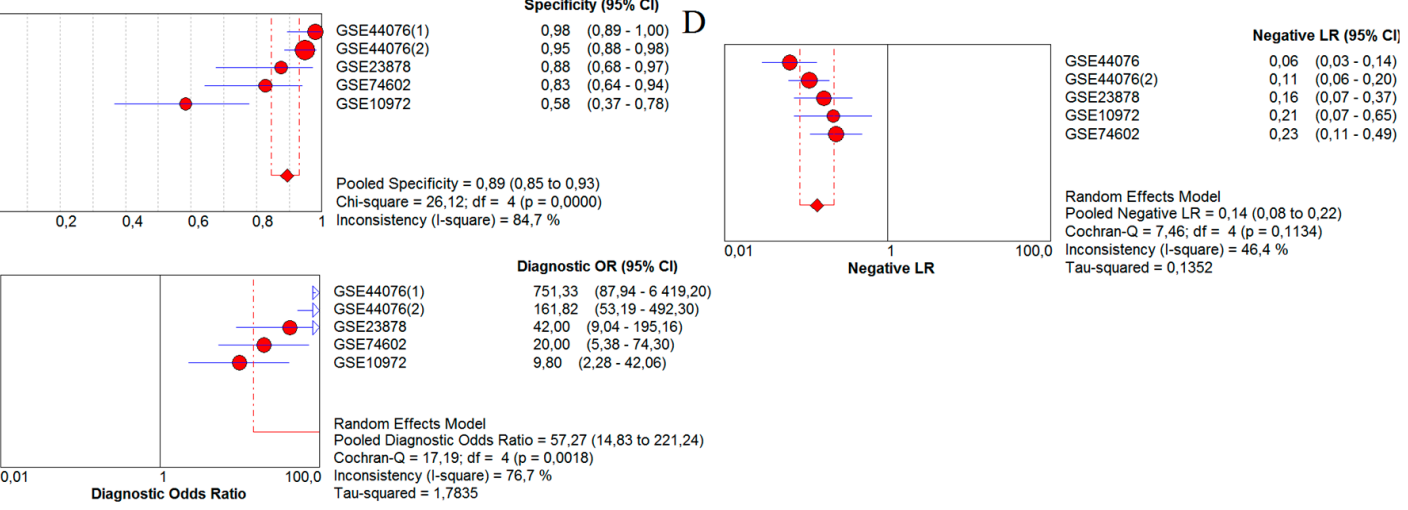

Figure 2. Forest plots of meta-analysis for CHGA as diagnostic biomarker in early-stage colon cancer. Different points represented various studies in the meta-analysis, which were arranged from high to low by effect size. (A) forest plot of sensitivity (0.89). By calculating the $\mathrm{I}^{2}(20.5 \%)$, no significant heterogeneity was found for this meta-analysis; (B) forest plot of specificity (0.89); (C) forest plot of positive-likelihood ratio (PLR) (7.86); (D) forest plot of negative-likelihood ratio (NLR) (0.14); (E) forest plot of diagnostic odds ratio (DOR) (57.27).

The summary receiver operator characteristic (SROC) curve for CHGA diagnostic meta-analysis is presented in Figure 3 and CHGA showed high diagnostic accuracy: area under curve (AUC) 0.9370 and $Q$ value 0.8736 . 


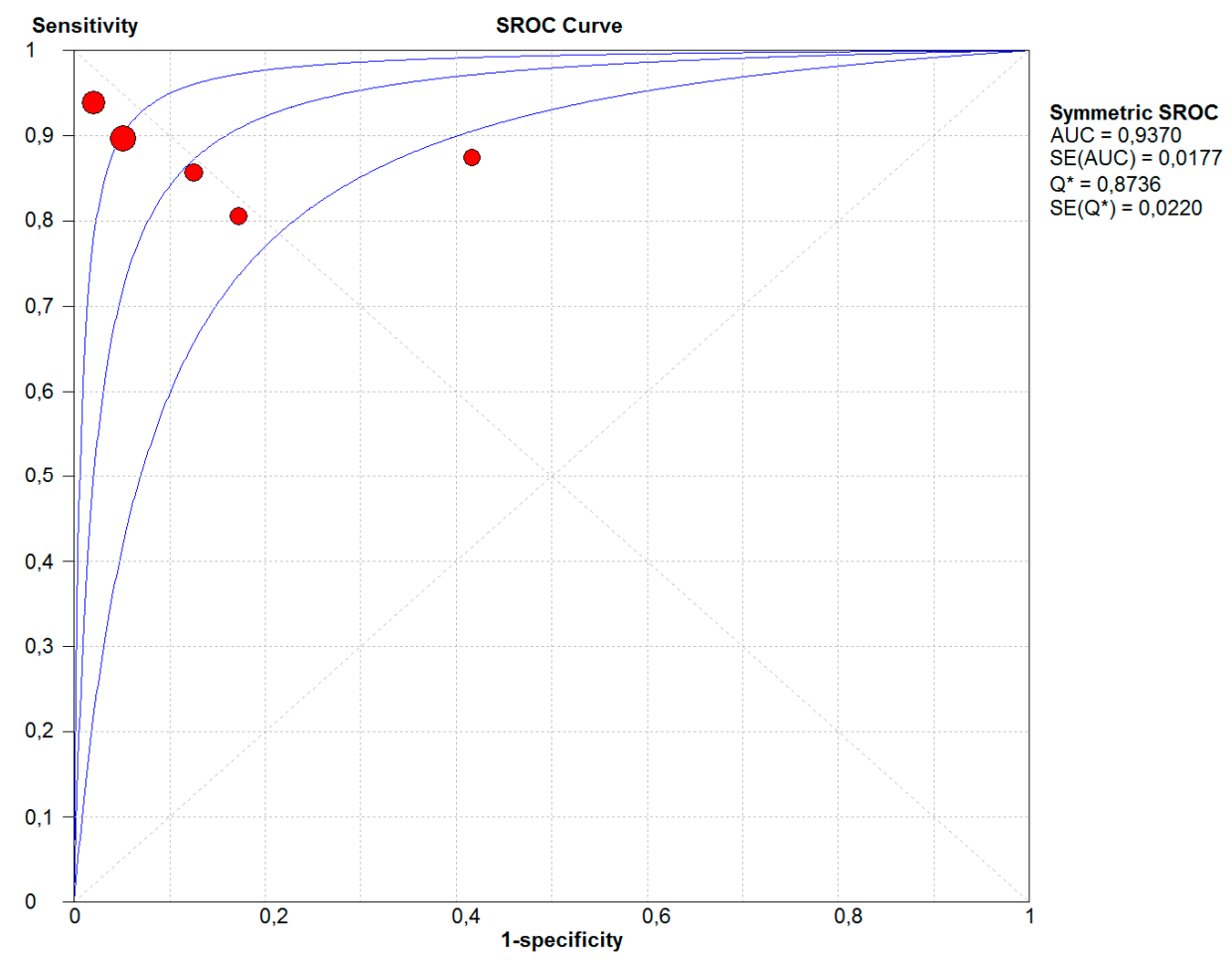

Figure 3. Summary receiver operator characteristic (SROC) curve for CHGA as an early diagnostic biomarker in colon cancer. Different points represent different studies in the meta-analysis and the size of points is the number of patients. There are three lines on the SROC curve: The middle line is the SROC curve fitted by the sensitivity (y-axis) and 1-specificity (x-axis) for corresponding studies, and the other two lines are the confidence interval. The SROC curve reflects diagnostic accuracy for biomarkers and the bigger size of area under curve (AUC) presents the better diagnostics accuracy for biomarkers. CHGA shows high diagnostic accuracy $(\mathrm{AUC}=0.9370)$ in colon cancer.

\subsection{Comparison of the CHGA with Other Biomarkers}

In order to make a comparison for the diagnostic effect of the CHGA with the identified biomarkers, the diagnostic meta-analysis for MKI67, TP53, and KRAS were conducted using the same datasets. The results for these meta-analyses are listed in Table 3.

Table 3. Comparison of CHGA with several typically identified biomarkers.

\begin{tabular}{lllllllll}
\hline Biomarker & Sensitivity & Specificity & PLR & NLR & DOR & AUC & Q Value & I $^{\mathbf{2 *}}$ \\
\hline CHGA & 0.89 & 0.89 & 46.94 & 0.14 & 57.27 & 0.9370 & 0.8736 & 0.205 \\
MKI67 & 0.86 & 0.81 & 4.60 & 0.18 & 27.65 & 0.9270 & 0.8615 & 0.527 \\
TP53 & 0.78 & 0.54 & 1.81 & 0.64 & 2.36 & 0.7732 & 0.7129 & 0.78 \\
KRAS & 0.82 & 0.77 & 2.83 & 0.30 & 9.78 & 0.8745 & 0.8050 & 0.812 \\
\hline
\end{tabular}

${ }^{*} \mathrm{Q}$ value: the point closest to the ideal top left-hand concer (sensitivity = specificity) on SROC curve; $\mathrm{I}^{2}$ : the measure for heterogeneity in meta-analysis.

\subsection{Verification in RNA-Seq Data}

The CHGA expression of the microarray datasets was verified in the RNA-seq data from GTEx and TCGA databases. Figure 4A-D presents the expression of the microarray datasets from our meta-analysis. Figure 4E shows the CHGA expression levels for colon cancer patients and healthy controls in TCGA and GTEX databases. The expression of the CHGA levels was markedly reduced in the colon cancer patients as compared with the CHGA expression in the normal controls in both microarray and RNA-seq data. 

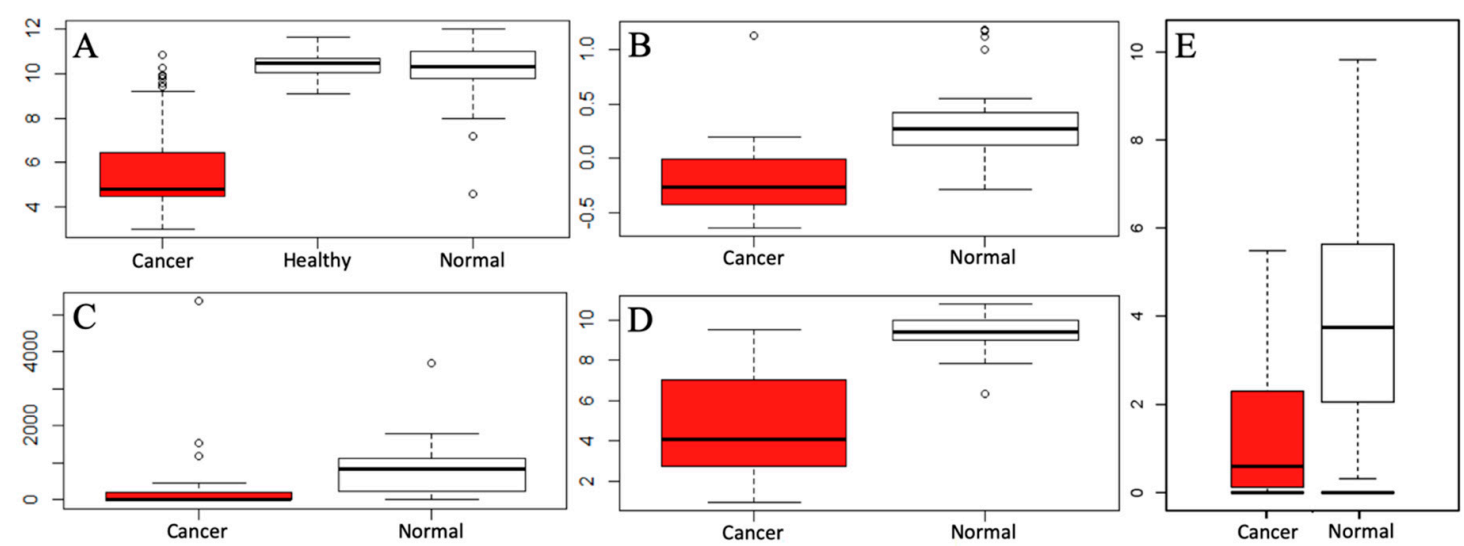

Cancer Normal

Figure 4. CHGA expression for colon cancer (in red solid box) and normal controls (in empty box) in microarray and RNA-seq data. (A) CHGA expression for colon cancer patients, healthy control, and normal adjacent mucosa of the colon cancer patients from the GSE44076 dataset; (B) CHGA expression for colon cancer patients and normal adjacent mucosa of the colon cancer patients from the GSE74602 dataset; (C) CHGA expression for colon cancer patients and normal adjacent mucosa of the colon cancer patients from the GSE10972 dataset; (D) CHGA expression for colon cancer patients and normal control of adjacent mucosa of the colon cancer patients from the GSE23878 dataset; (E) CHGA expression in colon cancer patients and normal controls from RNA-seq data from TCGA and GTEx.

\subsection{CHGA-Related PPI Networks and Biological Explanation}

Our previous study showed that CRC biomarkers had strong relationships on the PPI network [10]. Therefore, we supposed that the close neighbors with CHGA on the PPI network had high possibility to be further biomarkers. In order to further analyze the biological interactions for CHGA, PPI networks were constructed for both its closest proteins and other biomarkers in Figure 5. SCG3, SCG2, SST, NCAM1, ENO2, GAST, SYP, SYT1, STX1A, and CHGB, as the nearby proteins of the CHGA, were predicted as potential future early diagnostic biomarkers for colon cancer (Figure 5A). CHGA expression was found to be associated with KRAS and TP53 (Figure 5B).
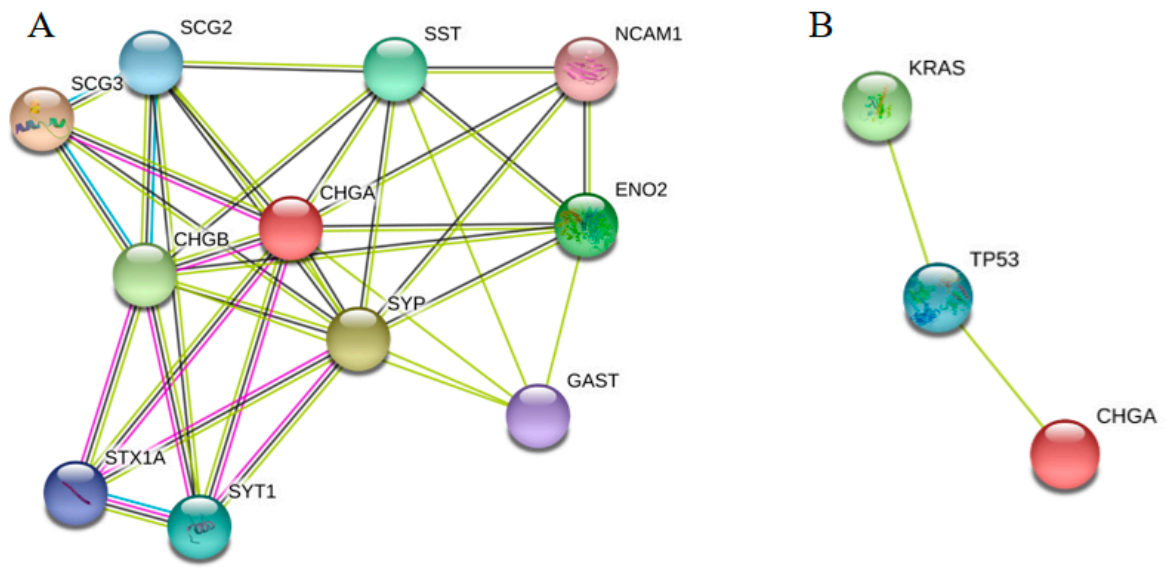

Figure 5. Closest PPI network for CHGA (A). The String database was utilized to draw the human PPI networks showing associations of CHGA with its closest neighbors. Relationships of CHGA, TP53, and KRAS (B). Several well-known biomarkers such as KRAS, TP53, and MKI67 were input together with CHGA in the String database, showing that TP53 had interaction relationships with CHGA and KRAS. Different points represented different proteins and different lines indicated the interactions from different evidences. Green lines: The evidence from neighborhood genes; Black line: The proteins co-expression; Purple line: The evidence from the experiment; Blue line: The evidence from curated databases. 
In order to investigate the biological explanation for the reason why CHGA performed so well in the diagnosis of early-stage colon cancer, gene ontology (GO) analyses were conducted for the CHGA-related genes (from closest PPI and CHGA-TP53-KRAS PPI, Table 4). We found that CHGA and its closest genes were strongly associated with the regulations of cell communication and signaling at the biological function level (Table 4A). At the cellular component level, they are closely linked to the transport functions (Table 4B). Additionally, we found that CHGA and its related biomarkers (TP53 and KRAS) were mapped on the regulation of neuron death and cell death pathways (Table 4C).

Table 4. Biological function analysis results for CHGA-related genes and biomarkers. (A) Biological function (GO) for CHGA closest genes. (B) GO for CHGA closest genes. (C) GO for CHGA and TP53, KRAS.

\begin{tabular}{|c|c|c|c|c|}
\hline \multicolumn{5}{|c|}{ (A) } \\
\hline GO ID & Pathway & Count & FDR $^{*}$ & Matching Genes \\
\hline GO:0010646 & $\begin{array}{l}\text { regulation of cell } \\
\text { communication }\end{array}$ & 9 & 0.0018 & $\begin{array}{l}\text { CHGA, CHGB, GAST, NCAM1, } \\
\text { SCG2, SST, STX1A, SYP, SYT1 }\end{array}$ \\
\hline GO:0023051 & regulation of signaling & 9 & 0.0018 & $\begin{array}{l}\text { CHGA, CHGB, GAST, NCAM1, } \\
\text { SCG2, SST, STX1A, SYP, SYT1 }\end{array}$ \\
\hline GO:0050433 & $\begin{array}{l}\text { regulation of catecholamine } \\
\text { secretion }\end{array}$ & 3 & 0.0018 & CHGA, STX1A, SYT1 \\
\hline GO:0048583 & $\begin{array}{l}\text { regulation of response to } \\
\text { stimulus }\end{array}$ & 9 & 0.0022 & $\begin{array}{l}\text { CHGA, CHGB, GAST, NCAM1, } \\
\text { SCG2, SST, STX1A, SYP, SYT1 }\end{array}$ \\
\hline GO:0009966 & regulation of signal transduction & 8 & 0.0028 & $\begin{array}{l}\text { CHGA, CHGB, GAST, NCAM1, } \\
\text { SCG2, SST, STX1A, SYP }\end{array}$ \\
\hline GO:0032940 & secretion by cell & 5 & 0.0064 & CHGA, SCG2, SCG3, STX1A, SYT1 \\
\hline GO:0070887 & $\begin{array}{l}\text { cellular response to chemical } \\
\text { stimulus }\end{array}$ & 7 & 0.0083 & $\begin{array}{l}\text { CHGA, NCAM1, SCG2, SST, } \\
\text { STX1A, SYP, SYT1 }\end{array}$ \\
\hline GO:0010469 & $\begin{array}{l}\text { regulation of signaling receptor } \\
\text { activity }\end{array}$ & 4 & 0.0084 & CHGB, GAST, SCG2, SST \\
\hline GO:0042221 & response to chemical & 8 & 0.0127 & $\begin{array}{l}\text { CHGA, GAST, NCAM1, SCG2, SST, } \\
\text { STX1A, SYP, SYT1 }\end{array}$ \\
\hline GO:0045055 & regulated exocytosis & 4 & 0.014 & CHGA, SCG3, STX1A, SYT1 \\
\hline \multicolumn{5}{|c|}{ (B) } \\
\hline GO ID & Pathway & Count & FDR $^{*}$ & Matching Genes \\
\hline GO:0030658 & transport vesicle membrane & 5 & $4.82 \times 10-^{6}$ & CHGA, SCG3, STX1A, SYP, SYT1 \\
\hline GO:0099503 & secretory vesicle & 7 & $1.33 \times 10-^{5}$ & $\begin{array}{l}\text { CHGA, CHGB, SCG2, SCG3, } \\
\text { STX1A, SYP, SYT1 }\end{array}$ \\
\hline GO:0030141 & secretory granule & 6 & $8.41 \times 10-^{5}$ & $\begin{array}{l}\text { CHGA, CHGB, SCG2, SCG3, } \\
\text { STX1A, SYT1 }\end{array}$ \\
\hline GO:0005576 & extracellular region & 8 & 0.00024 & $\begin{array}{l}\text { CHGA, CHGB, GAST, NCAM1, } \\
\text { SCG2, SCG3, SST, STX1A }\end{array}$ \\
\hline GO:0042583 & chromaffin granule & 2 & 0.00024 & CHGA, SYT1 \\
\hline GO:0098588 & $\begin{array}{l}\text { bounding membrane of } \\
\text { organelle }\end{array}$ & 6 & 0.0019 & $\begin{array}{l}\text { CHGA, NCAM1, SCG3, STX1A, } \\
\text { SYP, SYT1 }\end{array}$ \\
\hline GO:0012505 & endomembrane system & 8 & 0.003 & $\begin{array}{l}\text { CHGA, CHGB, NCAM1, SCG2, } \\
\text { SCG3, STX1A, SYP, SYT1 }\end{array}$ \\
\hline GO:0005615 & extracellular space & 4 & 0.0125 & CHGA, GAST, SCG2, SST \\
\hline \multicolumn{5}{|c|}{ (C) } \\
\hline GO ID & Pathway & Count & FDR $^{*}$ & Matching Genes \\
\hline GO:1901214 & regulation of neuron death & 3 & 0.0023 & CHGA, TP53, KRAS \\
\hline GO:0060548 & negative regulation of cell death & 3 & 0.0449 & CHGA, TP53, KRAS \\
\hline
\end{tabular}




\subsection{Prediction for CHGA-Related Biomarkers from Expression Levels}

The genes with similar expression levels always performed relatively similar functions [30]. Figure 6 shows the similar expressions of the genes to CHGA in colon cancer, which were predicted as further biomarker candidates.

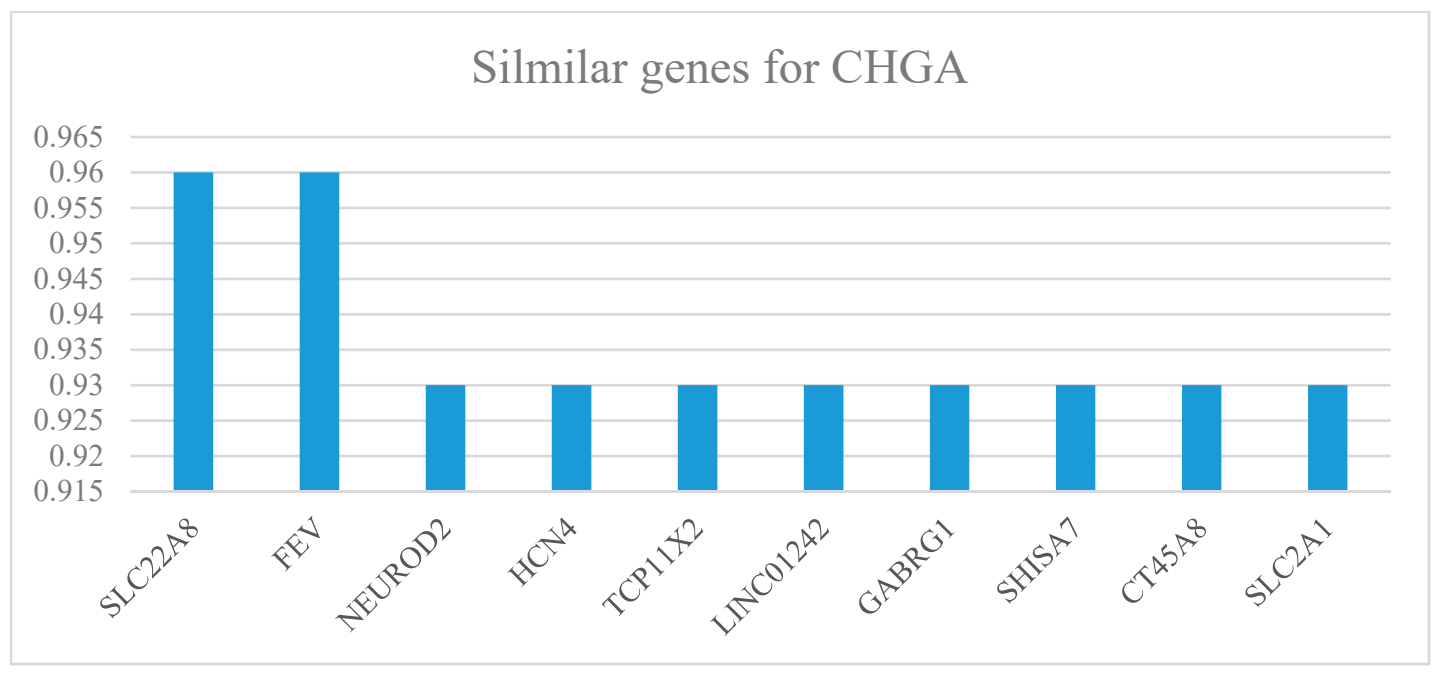

Figure 6. Similar genes that have similar expression patterns, ranked by Pearson correlation coefficients, for CHGA in colon cancer patients.

\section{Discussion}

Accumulating evidence has shown that the majority of colon cancer patients in their early stages (I and II) will benefit from modern cancer therapies, and their five-year survival rate has reached up to more than $90 \%$. However, the five-year survival rate for the later stages (III and IV) of colon cancer patients remain at about $10 \%$ [3]. Moreover, more than $50 \%$ of colon cancer patients are already at the late stages when their cancer is clinically diagnosed [4]. The strict rule is that the earlier the diagnosis for cancers, including colon cancer, the better therapies the cancer patients will receive and the better the outcome the patients will have. It; therefore, appears significantly important to search for more convenient, accurate, and powerful biomarkers to meet such an urgent need for the early diagnosis of colon cancer.

Biomarkers as biological indicators and conditions have been widely shown to improve the diagnosis, therapeutic response, and prognosis of colon cancer [5]. Many cancer researchers, including our CRC research group, have been working on identifying the significance of various biomarkers for CRC [7-10]. In order to study the essential roles and important functions of biomarkers in CRC, we have created a CRC biomarker database [11], and have further analyzed, with AI-assisted verification, the potential applications of DNA, RNA, and protein biomarkers in diagnosis, therapy, and prognosis for CRC [10].

CHGA is a 439-Kd protein in the secretory granules of many normal and neoplastic neuroendocrine cells and it plays an essential role in the mechanisms of protein storage and release [13]. CHGA was considered as a biomarker for neuroendocrine neoplasms $[17,18]$ and was approved, with microarrays and tissue arrays, as a potential biomarker for early cancer diagnosis of gastric cancer [19] and prostate cancer [20]. The majority of pancreatic neuroendocrine tumors showed CHGA positive immunostaining, and primary tumors with metastases revealed significantly less CHGA protein expression than primary tumors without metastases [21]. However, there is no study concerning CHGA in early diagnosis of colon cancer.

In this study, CHGA expression was found to be decreased in the early stages of colon cancer in patients, as compared to CHGA expression levels in both the healthy populations and the normal colon cancer mucosa from the colon cancer patients. This evidence indicated that CHGA 
might play an essential role in the initiation of colon cancer, from the normal colon tissue to early cancer. The down-expression of CHGA might be one of the critical mechanisms leading to colon cancer formation.

In one of our ongoing studies, CHGA expression was predicted as a promising early diagnosis biomarker for colon cancer via SVM and regression tree analysis, from the reported colon cancer diagnostic biomarkers' topology features on the PPI network [28], and the diagnostic value of CHGA expression in colon cancer was further verified. Meta-analysis, with its high scientific confidence, has been used to detect the diagnostic value of different biomarkers in various diseases [31,32]. The GEO database has been widely used in bioinformatics analysis because it is a comprehensive database storing big amounts of GE data. As such, more and more studies have been recently focused on the meta-analysis based on the GEO datasets, and a majority of the meta-analysis began with GE differential analyses $[33,34]$. Our results showed even higher confidence than the GE differential analyses, since we predicted CHGA expression as a candidate biomarker by machine learning (SVM and regression tree) based on the published biomarkers [28].

According to our previous study, CRC biomarkers always had strong relationships on the PPI networks [10]. Hence, we predicted several valuable biomarkers from the CHGA closest PPI network (Figure 5A) which deserved further verification. In order to detect the functions and applications of these closest neighbors of the CHGA, literature verifications were performed. We searched relevant published papers from PubMed and Google scholar concerning these nearby proteins and cancers, and found that SCG3 was convinced as a prognostic biomarker for lung cancer [35] and rectal cancer [36]; SST expression was reported to have a strong relationship with advanced CRC [37]; NCAM1 was correlated with several human cancers [38]; ENO2 were predicted as treatment biomarker for acute lymphoblastic leukemia [39]; SYT1 expression was a candidate colon cancer biomarker [40]; STAX1 was proved to be associated with the transformation of high-grade tumors in the bladder cancer [41]; and as a paralog for CHGA, CHGB was a prognostic marker for the pancreatic neuroendocrine tumors [21]. Expression of SLC22A8 was considered as a valuable biomarker for the lung cancer treatment [42]. The NEUROD2 gene was related to the metastasis and the survival for colon cancer [43]. The SLC2A1 gene was related to the metabolic shift of colon cancer [44], and the HCN4 gene was correlated with low survival rate of multiple cancers [45].

A variety of biomarkers, as biological indicators in their pathways, have been widely used to improve the cancer diagnosis, therapeutic response, and prognosis, including for colon cancer [6]. A majority of cancer researchers, including our CRC research group, have been focusing on the typical oncogenes, such as KRAS [22], and classic tumor suppressors like TP53 [23], to clarify their significance of various biomarkers in colon cancer. KRAS, as a typical oncogene to initiate cancer development, has been proven in many cancer types, especially in CRC, with strong early diagnosis impact, even in the cancer progression and prognosis [22]. There are more than $50 \%$ of CRC patients with KRAS gene mutations in their early stages, and half of such patient are not benefiting from antibody therapy [22]. TP53, as a classic tumor suppressor, has revealed more than $60 \%$ of CRC patients with TP53 mutations, which is one of the commonest genetic events in the development of human CRC [23]. MKI67 is a biomarker of cellular proliferation and has been reported as an independent index for CRC cell growth [22]. The expression of MKI67 has been gradually increased from normal tissue, adenomas to adenocarcinomas, in CRC patients. The overexpression of MKI67 has been considered as the significant biomarker and predictor for primary CRC screening [25].

Majority of the candidate biomarkers predicted based on CHGA PPI and CHGA similar genes were further verified through the literatures studies. We showed that the candidate biomarkers were associated with the diagnosis, therapy, or prognosis of human cancers, including colon cancer, in which provided further evidence for the CHGA as a potential hub biomarker for the colon cancer. Furthermore, we found several important pathways for the CHGA and its closest PPI neighbors, as well as other biomarkers from GO annotation: The regulation of cell communication and signaling at the biological function level, the transport function, and the regulation of neuron death and cell 
death, which should be important for future colon cancer biomarker discovery. In the near future, there will be more practical machine learning techniques that will be introduced to the field of precise biomarker discovery, for the identification and verification of potential biomarkers for early diagnosis, therapy response, and prognosis in cancers.

\section{Materials and Methods}

\subsection{Data Collection, Extraction, and Normalization}

We followed the "Preferred Reporting Items for Systematic Reviews and Meta-Analyses" guidelines to conduct this meta-analysis.

Two investigators independently searched the GEO database for relevant studies up to February 2019. The following search terms were utilized: colon AND (cancer OR carcinoma OR neoplasia). We recorded the homo sapiens relevant series as a further filter.

The criteria to select the needed studies was as following: (1) The study contains the GE data for early-stage colon cancer patients and normal healthy controls. (2) There are clear descriptions for the patients' situation and experiment methods. The datasets recorded the country, number, and source of included samples, and the experiment methods and platform were clearly introduced, for example: "Expression profiling by array," platform "GPL13667." (3) The suitable dataset should contain at least 30 samples. (4) The dataset should include the GE data for CHGA. R package "GEOquery" was used to download the GE data from the GEO database, and the data were standardized to log scale. The function "normalizeQuantiles" in "Limma" $\mathrm{R}$ package was utilized to further normalize the log-scaled GE data.

\subsection{Logistic Regression and Diagnostic Meta-Analysis}

We extracted the GE data of CHGA for both the cancer group and the control group, and implemented logistic regression analysis to get true positive (TP), false positive (FP), true negative (TN), and false negative (FN) results for diagnostic meta-analysis. In this logistic regression, GE level was used as the variable.

Diagnostic meta-analysis for early-stage colon cancer was conducted, and a random effects model was selected as a standard statistical method in this study, which did not need new data to train the models and allowed the differences among the different studies.

The statistical data were as follows: sensitivity, specificity, PLR, NLR, and DOR with corresponding 95\% confidence intervals. The SROC curves were plotted based on the sensitivity and specificity. A random effect model was used for the statistics. The heterogeneity among studies was assessed by $\mathrm{I}^{2}$ on sensitivity of the CHGA diagnostic test. $\mathrm{I}^{2}<50 \%$ was considered as small heterogeneity.

\subsection{Verification Test}

In order to verify the meta-analysis result, we used RNA-seq data from the TCGA and GTEx database to compare the CHGA expression level between colon cancer patients with healthy samples. Meanwhile we also drew the boxplot for CHGA expression in our collected microarray data.

\subsection{CHGA PPI Network Construction, Biological Function Analysis, and Detection for CHGA Similar Genes}

We drew the PPI network for searching for the neighbors for CHGA based on protein interaction evidence, and investigated the PPI relationships of CHGA with some reported biomarkers (TP53, KRAS, and MKI67). GO annotation was conducted for analyzing the biological functions of the CHGA-related genes and biomarkers. The genes with similar expression were considered as similar genes, and we used Pearson correlation coefficients to calculate the similar genes for CHGA to provide evidence for further prediction of new biomarkers. 


\subsection{Software and Tools}

$\mathrm{R}$ language was used to download the GEO data and make normalization, and logistic regression was conducted by SPSS 22.0. Diagnostic meta-analysis and heterogeneity analysis were implemented by MetaDisc 1.4. Verification test and similar genes detection were executed on the Gene Expression Profiling Interactive Analysis (GEPIA) database. The String database was used to draw the PPI network and conduct the biological function analyses.

\section{Conclusions}

A logistic regression-based meta-analysis was used to analyze the significant roles and functions of CHGA expression as a novel and promising significant biomarker for early diagnosis of colon cancer patients, and to compare CHGA in early diagnosis with the well-known oncogene, KRAS, tumor suppressor, TP53, and cellular proliferative factor, MKI67, in colon cancer. CHGA might be a further biomarker for early colon cancer patients. Several other biomarkers from the PPI network, and similar genes to CHGA, were further predicted for future early diagnosis of colon cancer.

Author Contributions: Conceptualization, X.Z. and X.-F.S.; data curation, X.Z.; formal analysis, X.Z.; investigation, X.Z.; methodology, X.Z.; software, X.Z.; supervision, X.-F.S., B.S. and H.Z.; visualization, X.Z. and X.-F.S.; writing—original draft, X.Z.; writing—review and editing X.-F.S.

Funding: This study was financially supported by the Swedish Cancer Foundation and the Swedish Research Council.

Acknowledgments: The authors are grateful to the staff in our research groups, who were involved in the study, for their valuable contributions and discussions. The authors thank Dirk Repsilber for his help in the machine learning knowledge, and they also thank Ming Min and Cheng Qian for their help in figure and linguistics revision.

Conflicts of Interest: The authors declare no conflicts of interest.

\section{References}

1. Siegel, R.L.; Miller, K.D.; Jemal, A. Cancer statistics, 2019. Cancer J. Clin. 2019, 69, 7-34. [CrossRef] [PubMed]

2. Bray, F.; Ferlay, J.; Soerjomataram, I.; Siegel, R.L.; Torre, L.A.; Jemal, A. Global cancer statistics 2018: GLOBOCAN estimates of incidence and mortality worldwide for 36 cancers in 185 countries. Cancer J. Clin. 2018, 68, 394-424. [CrossRef] [PubMed]

3. Brenner, H.; Kloor, M.; Pox, C.P. Colorectal cancer. Lancet 2014, 383, 1490-1502. [CrossRef]

4. Siegel, R.L.; Miller, K.D.; Fedewa, S.A.; Ahnen, D.J.; Meester, R.G.S.; Barzi, A.; Jemal, A. Colorectal cancer statistics, 2017. Cancer J. Clin. 2017, 67, 177-193. [CrossRef] [PubMed]

5. Geiger, T.M.; Ricciardi, R. Screening options and recommendations for colorectal cancer. Clin. Colon Rectal Surg. 2009, 22, 209-217. [CrossRef] [PubMed]

6. Newton, K.F.; Newman, W.; Hill, J. Review of biomarkers in colorectal cancer. Colorectal Dis. 2012, $14,3-17$. [CrossRef] [PubMed]

7. Sun, X.F.; Carstensen, J.M.; Zhang, H.; Stal, O.; Wingren, S.; Hatschek, T.; Nordenskjold, B. Prognostic-significance of cytoplasmic-P53 oncoprotein in colorectal adenocarcinoma. Lancet 1992, 340, 1369-1373. [CrossRef]

8. Sun, X.F.; Ekberg, H.; Zhang, H.; Carstensen, J.M.; Nordenskjold, B. Overexpression of ras is an independent prognostic factor in colorectal adenocarcinoma. APMIS 1998, 106, 657-664. [CrossRef] [PubMed]

9. Sun, X.F.; Bartik, Z.; Zhang, H. Bcl-2 expression is a prognostic factor in the subgroups of patients with colorectal cancer. Int. J. Oncol. 2003, 23, 1439-1443. [CrossRef] [PubMed]

10. Zhang, X.; Sun, X.F.; Shen, B.; Zhang, H. Potential applications of DNA, RNA and protein biomarkers in diagnosis, therapy and prognosis for colorectal cancer: A study from databases to AI-assisted verification. Cancers 2019, 11, 172. [CrossRef]

11. Zhang, X.; Sun, X.F.; Cao, Y.; Ye, B.; Peng, Q.; Liu, X.; Shen, B.; Zhang, H. CBD: A biomarker database for colorectal cancer. Database 2018. [CrossRef] [PubMed] 
12. Takiyyuddin, M.A.; Cervenka, J.H.; Sullivan, P.A.; Pandian, M.R.; Parmer, R.J.; Barbosa, J.A.; O'Connor, D.T. Is physiologic sympathoadrenal catecholamine release exocytotic in humans? Circulation 1990, 81, $185-195$. [CrossRef] [PubMed]

13. Mahapatra, N.R.; O'Connor, D.T.; Vaingankar, S.M.; Hikim, A.P.; Mahata, M.; Ray, S.; Staite, E.; Wu, H.; $\mathrm{Gu}$, Y.; Dalton, N.; et al. Hypertension from targeted ablation of chromogranin A can be rescued by the human ortholog. J. Clin. Investig. 2005, 115, 1942-1952. [CrossRef] [PubMed]

14. Kleist, B.; Poetsch, M. Neuroendocrine differentiation: The mysterious fellow of colorectal cancer. World J. Gastroenterol. 2015, 21, 11740-11747. [CrossRef] [PubMed]

15. Zeng, Y.J.; Lai, W.; Liu, L.; Wu, H.; Luo, X.X.; Wang, J.; Chu, Z.H. Prognostic significance of neuroendocrine differentiation in colorectal adenocarcinoma after radical operation: A meta-analysis. J. Gastrointest Surg. 2014, 18, 968-976. [CrossRef] [PubMed]

16. Dost Gunay, F.S.; Kirmizi, B.A.; Ensari, A.; Icli, F.; Akbulut, H. Tumor-associated macrophages and neuroendocrine differentiation decrease the efficacy of bevacizumab plus chemotherapy in patients with advanced colorectal cancer. Clin. Colorectal Cancer 2018, 18, e244-e250. [CrossRef]

17. Oberg, K. Biochemical diagnosis of neuroendocrine GEP tumor. Yale J. Biol. Med. 1997, 70, 501-508.

18. Tomassetti, P.; Migliori, M.; Simoni, P.; Casadei, R.; De Iasio, R.; Corinaldesi, R.; Gullo, L. Diagnostic value of plasma chromogranin A in neuroendocrine tumours. Eur. J. Gastroenterol. Hepatol. 2001, 13, 55-58. [CrossRef]

19. Yang, S.; Chung, H.C. Novel biomarker candidates for gastric cancer. Oncol. Rep. 2008, 19, 675-680. [CrossRef]

20. Ma, Z.; Tsuchiya, N.; Yuasa, T.; Huang, M.; Obara, T.; Narita, S.; Horikawa, Y.; Tsuruta, H.; Saito, M.; Satoh, S.; et al. Clinical significance of polymorphism and expression of chromogranin A and endothelin-1 in prostate cancer. J. Urol. 2010, 184, 1182-1188. [CrossRef]

21. Weisbrod, A.B.; Zhang, L.; Jain, M.; Barak, S.; Quezado, M.M.; Kebebew, E. Altered PTEN, ATRX, CHGA, CHGB, and TP53 expression are associated with aggressive VHL-associated pancreatic neuroendocrine tumors. Horm. Cancer 2013, 4, 165-175. [CrossRef] [PubMed]

22. Karapetis, C.S.; Khambata-Ford, S.; Jonker, D.J.; O'Callaghan, C.J.; Tu, D.; Tebbutt, N.C.; Simes, R.J.; Chalchal, H.; Shapiro, J.D.; Robitaille, S.; et al. K-ras mutations and benefit from cetuximab in advanced colorectal cancer. N. Engl. J. Med. 2008, 359, 1757-1765. [CrossRef] [PubMed]

23. Rodrigues, N.R.; Rowan, A.; Smith, M.E.F.; Kerr, I.B.; Bodmer, W.F.; Gannon, J.V.; Lane, D.P. P53 mutations in colorectal cancer. Proc. Natl. Acad. Sci. USA 1990, 87, 7555-7559. [CrossRef] [PubMed]

24. Yang, Y.C.; Li, J.; Jin, L.; Wang, D.; Zhang, J.H.; Wang, J.; Zhao, X.M.; Wu, G.C.; Yao, H.W.; Zhang, Z.T. Independent correlation between Ki67 index and circulating tumor cells in the diagnosis of colorectal cancer. Anticancer Res. 2017, 37, 4693-4700. [CrossRef] [PubMed]

25. Heidari, Z.; Mahmoudzadeh-Sagheb, H.; Jahantigh, M.; Gorgich, E.A.C. Immunohistochemical expression of Ki67 and HER2 in colorectal cancer compared to adenomatous and normal samples. Int. J. Cancer Manag. 2017, 10. [CrossRef]

26. Larranaga, P.; Calvo, B.; Santana, R.; Bielza, C.; Galdiano, J.; Inza, I.; Lozano, J.A.; Armananzas, R.; Santafe, G.; Perez, A.; et al. Machine learning in bioinformatics. Brief. Bioinform. 2006, 7, 86-112. [CrossRef] [PubMed]

27. Szklarczyk, D.; Franceschini, A.; Wyder, S.; Forslund, K.; Heller, D.; Huerta-Cepas, J.; Simonovic, M.; Roth, A.; Santos, A.; Tsafou, K.P.; et al. STRING v10: Protein-protein interaction networks, integrated over the tree of life. Nucleic Acids Res. 2015, 43, D447-D452. [CrossRef] [PubMed]

28. Zhang, X.; Sun, X.F.; Shen, B.; Zhang, H. Machine learning based biomarker discovery for colon cancer from protein-protein interaction network. (unpublished, manuscript in preparation).

29. Moher, D.; Liberati, A.; Tetzlaff, J.; Altman, D.G.; Group, P. Preferred reporting items for systematic reviews and meta-analyses: The PRISMA statement. PLoS Med. 2009, 6, e1000097. [CrossRef]

30. Tang, Z.F.; Li, C.W.; Kang, B.X.; Gao, G.; Li, C.; Zhang, Z.M. GEPIA: A web server for cancer and normal gene expression profiling and interactive analyses. Nucleic Acids Res. 2017, 45, W98-W102. [CrossRef]

31. Peng, Q.; Zhang, X.; Min, M.; Zou, L.; Shen, P.; Zhu, Y. The clinical role of microRNA-21 as a promising biomarker in the diagnosis and prognosis of colorectal cancer: A systematic review and meta-analysis. Oncotarget 2017, 8, 44893-44909. [CrossRef]

32. Xu, L.H.; Guo, Y.; Zhang, X.; Chen, J.J.; Hu, S.Y. Blood-based circulating microRNAs are potential diagnostic biomarkers for leukemia: Result from a meta-analysis. Cell. Physiol. Biochem. 2016, 38, 939-949. [CrossRef] [PubMed] 
33. Wang, Y.M.; Xue, D.; Li, Y.W.; Pan, X.Y.; Zhang, X.Y.; Kuang, B.; Zhou, M.; Li, X.L.; Xiong, W.; Li, G.Y.; et al. The Long Noncoding RNA MALAT-1 is a novel biomarker in various cancers: A meta-analysis based on the GEO database and literature. J. Cancer 2016, 7, 991-1001. [CrossRef] [PubMed]

34. Long, N.P.; Jung, K.H.; Anh, N.H.; Yan, H.H.; Nghi, T.D.; Park, S.; Yoon, S.J.; Min, J.E.; Kim, H.M.; Lim, J.H.; et al. An integrative data mining and omics-based translational model for the identification and validation of oncogenic biomarkers of pancreatic cancer. Cancers 2019, 11, 155. [CrossRef] [PubMed]

35. Moss, A.C.; Jacobson, G.M.; Walker, L.E.; Blake, N.W.; Marshall, E.; Coulson, J.M. SCG3 transcript in peripheral blood is a prognostic biomarker for REST-deficient small cell lung cancer. Clin. Cancer Res. 2009, 15, 274-283. [CrossRef] [PubMed]

36. Sun, M.; Sun, T.; He, Z.; Xiong, B. Identification of two novel biomarkers of rectal carcinoma progression and prognosis via co-expression network analysis. Oncotarget 2017, 8, 69594-69609. [CrossRef] [PubMed]

37. Vuaroqueaux, V.; Dutour, A.; Briard, N.; Monges, G.; Grino, M.; Oliver, C.; Ouafik, L. No loss of sst receptors gene expression in advanced stages of colorectal cancer. Eur. J. Endocrinol. 1999, 140, 362-366. [CrossRef]

38. Crnic, I.; Strittmatter, K.; Cavallaro, U.; Kopfstein, L.; Jussila, L.; Alitalo, K.; Christofori, G. Loss of neural cell adhesion molecule induces tumor metastasis by up-regulating lymphangiogenesis. Cancer Res. 2004, 64, 8630-8638. [CrossRef]

39. Liu, C.C.; Wang, H.; Wang, W.D.; Wang, L.; Liu, W.J.; Wang, J.H.; Geng, Q.R.; Lu, Y. ENO2 Promotes Cell Proliferation, Glycolysis, and Glucocorticoid-Resistance in Acute Lymphoblastic Leukemia. Cell. Physiol. Biochem. 2018, 46, 1525-1535. [CrossRef]

40. Zhu, H.; Wu, T.C.; Chen, W.Q.; Zhou, L.J.; Wu, Y.; Zeng, L.; Pei, H.P. Screening for differentially expressed genes between left- and right-sided colon carcinoma by microarray analysis. Oncol. Lett. 2013, 6, 353-358. [CrossRef]

41. Raja, S.A.; Abbas, S.; Shah, S.T.A.; Tariq, A.; Bibi, N.; Yousuf, A.; Khawaja, A.; Nawaz, M.; Mehmood, A.; Khan, M.J.; et al. Increased expression levels of syntaxin 1A and synaptobrevin 2/vesicle-associated membrane protein-2 are associated with the progression of bladder cancer. Genet. Mol. Biol. 2019, 42, 40-47. [CrossRef]

42. Ooi, A.T.; Gomperts, B.N. Molecular pathways: Targeting cellular energy metabolism in cancer via inhibition of SLC2A1 and LDHA. Clin. Cancer Res. 2015, 21, 2440-2444. [CrossRef] [PubMed]

43. Zhou, Y.; Zang, Y.; Yang, Y.; Xiang, J.; Chen, Z. Candidate genes involved in metastasis of colon cancer identified by integrated analysis. Cancer Med. 2019, 8, 2338-2347. [CrossRef] [PubMed]

44. Santasusagna, S.; Moreno, I.; Navarro, A.; Munoz, C.; Martinez, F.; Hernandez, R.; Castellano, J.J.; Monzo, M. miR-328 mediates a metabolic shift in colon cancer cells by targeting SLC2A1/GLUT1. Clin. Transl. Oncol. 2018, 20, 1161-1167. [CrossRef] [PubMed]

45. Phan, N.N.; Huynh, T.T.; Lin, Y.C. Hyperpolarization-activated cyclic nucleotide-gated gene signatures and poor clinical outcome of cancer patient. Transl. Cancer Res. 2017, 6, 698-708. [CrossRef] 